\title{
RECONSTRUYENDO LA HISTORIA DE UNA COMUNIDAD PLANTACIONISTA: LA VASSOURAS DE STANLEY STEIN'
}

\section{RECONSTRUCTING THE HISTORY OF A PLANTATION COMMUNITY: STANLEY STEIN'S VASSOURAS}

\author{
Dale TOMich \\ Fernand Braudel Center \\ Binghamton University
}

\section{RESUMEN}

Publicado en 1957, Vassouras de Stanley Stein sigue siendo una obra esencial para la historia del Brasil y para los estudios sobre la plantación. En este artículo ofrecemos un análisis microhistórico de las diferentes fuentes intelectuales transdisciplinares que Stein movilizó para construir Vassouras en una época anterior a la existencia de la historia social. Es más, se enfatiza la forma en que hizo uso de los community studies para configurar una análisis que opera en múltiples escalas espaciales y temporales en un momento en que las aproximaciones microhistóricas no se habían desarrollado.

Palabras clave: Vassouras, esclavitud, Brasil, community studies.

\begin{abstract}
Published in 1957, Stanley Stein's Vassouras remains a class of Brazilian history and of plantation studies. This article offers a microhistorical analysis of the various transdisciplinary intellectual resources that Stein mobilized to construct Vassouras in a period before the existence of social history. Further, it emphasizes the way that utilized community studies to construct an analysis that operates on multiple spatial and temporal scale in a period when microhistorical approaches were not available.
\end{abstract}

Keywords: Vassouras, slavery, Brazil, community studies.

1 Una versión inicial de este artículo fue presentada en el coloquio Século XIX e as Novas Fronteiras da Escravidão e Liberdade, organizado en honor de Stanley y Barbara Stein en Río de Janeiro y Vassouras-R, Brasil, 11-14 de agosto de 2009. Se publicó como TOMICH (2016). 
A lo largo de más de cincuenta años la obra de Stanley Stein Vassouras: A Brazilian Coffee County, 1850-1900 no sólo ha llegado a ser una obligada referencia para la historia de la esclavitud y el café en Brasil sino que, además, se ha convertido en un clásico de la historiografía tanto brasileña como estadounidense. ${ }^{2}$ De hecho podemos decir que sigue siendo uno de los mejores estudios sobre la comunidad esclavista de plantación. Esta relevancia vigente de Vasssouras resulta si cabe más extraordinaria dado que fue escrita en un clima intelectual y académico muy distinto al de hoy en día. Como estudiante de doctorado en Harvard la audacia de Stein fue ir más allá de la historia narrativa focalizada en la política que se basaba en fuentes convencionales. Por el contrario, incorporó en su trabajo los últimos planteamientos de las ciencias sociales y la historiografía. Es más, el libro fue escrito antes que tuviera lugar la gran innovación historiográfica que comenzó en la década de 1960 con el consiguiente desarrollo en el campo de la Historia y las Ciencias sociales. Tomando como referente el nivel de este desarrollo las herramientas conceptuales de las que disponía eran extremadamente restringidas. Sin embargo, considero que es justo afirmar que el valor subsistente de Vassouras reside en el modo de reflexión con que Stein se aproximó a la construcción de su proyecto. La originalidad de su concepción y de sus innovadoras estrategias de análisis dio pie a aproximaciones muy útiles a los problemas conceptuales y metodológicos, que todavía hoy seguimos afrontando los historiadores, y anticipó los ulteriores debates en las ciencias sociales históricas.

2 En su origen una Tesis doctoral, el libro fue publicado por primera vez en 1957 por Harvard University Press. La primera edición brasileña apareció en 1961 en Editora Brasiliense con el título de Grandeza e Decadência do Café. La versión de 1957 se reimprimió en 1970 por Atheneum dentro de su colección "Studies in American Negro Life". En 1985 Princeton University Press publicó una edición conmemorativa con un nuevo prefacio del autor y 30 imágenes, incluyendo iconografía del siglo XIX y fotos inéditas del propio Stein que realizó durante su periodo de trabajo de campo. Por último, en 1990 Nova Fronteira publicó la traducción portuguesa de la última edición brasileña. 
Para apreciar mejor la riqueza historiográfica del trabajo de Stein me gustaría enfatizar alguna de las influencias intelectuales que podemos encontrar tanto en la conceptualización del proyecto como en su ejecución y que contribuyeron decisivamente a la solidez de la obra. Resulta especialmente importante realizar un análisis de estas influencias ya que, además del carácter aparentemente descriptivo de la obra, en las páginas de su estudio no encontraremos una teoría explícita. No son pocos los que consideran que las perspectivas analíticas sobre las que Stein articuló su proyecto están pasadas de moda. Pero una lectura atenta de cómo Stein estructuró su Vassouras nos revela que la obra todavía contiene un gran valor para la reflexión histórica en nuestros días.

\section{El MARCO INTELECTUAL: LA HISTORIA Y LAS CIENCIAS SOCIALES}

El propio título del libro, Vassouras: A Brazilian Coffee County, 18501900, ya nos dirige la atención hacia los elementos clave de su marco teórico y analítico. En primer lugar identifica el objeto de estudio como el municipio de Vassouras. El libro se concibe como el análisis de una comunidad. El término county dibuja la unidad de análisis de Stein y determina la arquitectura de la obra. Es decir, el libro no se construye alrededor de las relaciones entre amos y esclavos sino que más bien lo hace alrededor de un marco de relaciones más amplio entre todos los grupos que forman esta comunidad. El interés de Stein por el municipio de Vassouras nos permite ver la influencia de los community studies que tanto peso tuvieron en el ámbito de las Ciencias sociales en los Estados Unidos en las décadas de 1940 y 1950 y que inspiró la construcción del proyecto de investigación. En una sugerente entrevista mantenida con el historiador brasileño José Carlos Sebe Bom Meihy a finales de los 80 Stein subrayaba que Vassouras intentó ser una contribución a los community studies: "Por tanto, considero que resulta apropiado suponer que mi trabajo se integra en la tendencia historiográfica general que se desarrolló en Norteamérica alrededor de los debates técnicos y metodológicos en los community studies" (MEIHY, 1990: 85).

La importancia de los community studies en la obra de Stanley Stein es prueba de su abierta aproximación interdisciplinar al estudio histórico, incluso cuando era un simple estudiante de grado. Stein, un graduado del City College de New York en 1941, comenzó a estudiar historia de América Latina en el Departamento de Historia de la Universidad de Harvard bajo la dirección de Charles Haring después de haber servido en la Marina durante la Segunda Guerra Mundial. En la entrevista con Meihy, Stein recuerda: "Mientras todavía era estudiante en Harvard decidí no formarme 
sólo en historia política. Los cursos más importantes para mí fueron en antropología y sociología" (MEIHY, 1990: 84).

(El otro curso que resultó esencial para él fue el de Arthur Schlesinger sobre las ciudades norteamericanas en el que se enfatizaba la importancia de la cultura material en el desarrollo histórico). ${ }^{3}$ El interés de Stein en las ciencias sociales le permitió concebir la Historia como algo que estaba abierto a influencias interdisciplinares. Esta perspectiva le posibilitó ir más lejos de lo que la historia política predominante de aquel momento ofrecía para involucrarse en la historia económica y social.

En el diálogo que Stein mantiene con las ciencias sociales la antropología juega un papel fundamental. Por aquel entonces los community studies tenían una fuerte presencia en esta disciplina y ofrecían modelos de análisis útiles, especialmente para los estudiantes latinoamericanistas. Stein recuerda: "En aquel tiempo la Antropología era un pilar sólido de los community studies y había desarrollado técnicas que permitieron alcanzar resultados alentadores [...]. Además, por lo que respecta a los community studies, existía un número significativo de excelentes trabajos hechos sobre México que eran fruto de la investigación hecha en ese país desde la $\operatorname{Re}-$ volución mexicana. Algunos de estos trabajos eran obra de autores como Ralph Beals, Robert Redfield y, el último miembro de aquel grupo, Oscar Lewis [...]. Seguir la evolución metodológica de este grupo fue lo que me sugirió la forma de estudiar el caso de Brasil" (MEIHY, 1990: 84).

En palabras de Stein, "la antropología operaba como una vía de introducción a la historia social" (MEIHY, 1990: 84). (Vale la pena recordar que se formó como historiador varias décadas antes de la revolución de la historia social que tendría lugar en los 60 y 70). Al mismo tiempo, siguió de cerca el desarrollo metodológico de la investigación antropológica sobre las comunidades mexicanas para configurar su proyecto de historia social. La antropología le ofrecía no sólo un nuevo objeto de estudio sino también una nueva aproximación metodológica. Influenciado por antropólogos como Beals, Redfield y Lewis, definió el objeto de su investigación como la historia de una micro-región brasileña, una forma de estudiar Brasil que no era común en aquel momento.

La delimitación de la escala de análisis a una micro-región particular ofrecía una serie de ventajas prácticas obvias para una Tesis basada en fuentes primarias. Stein recuerda que: "Siguiendo este camino, esto es,

3 Stanley Stein reflexiona sobre sus experiencias antes de comenzar sus estudios de Historia en Harvard en MEIHY, 1990: 81-82. 
tomando una comunidad como mi punto de partida, no era necesario elaborar una tesis monumental, una explicación gigantesca de un país entero, ni tampoco de un continente, y cuanto menos esto era algo prudente para un extranjero que se iniciaba en el campo de los estudios brasileños" (MElHY, 1990: 84).

Por el contrario, esta estrategia le permitió elaborar un análisis profundo sobre temas diversos en archivos locales en el periodo de tiempo disponible para un joven investigador. Desde los inicios de su proyecto Stein perseguía romper con el uso de fuentes generales como los relatos de los viajeros, que eran tan comunes en la historiografía brasileña. La disponibilidad de fuentes locales era un factor fundamental en la elección de Vassouras como centro de su investigación. ${ }^{4}$ Como Stein recuerda: "no estaba interesado en recurrir al mismo tipo de fuentes como los relatos de viajes que siempre derivaban en análisis generales [...]. Esta práctica, usada desde el siglo XIX, ya había sido completamente trabajada, casi siempre produciendo historias genéricas y repetitivas. Quería hacer algo nuevo. Mi reto era basarme en documentación importante y combinarla con la memoria viva de las personas de aquel tiempo. Buscaba un puente entre la historia documentada y las experiencias que todavía podían ser aprehendidas" (MEIHY, 1990: 87). De hecho, Vassouras se distingue por su exploración vanguardista de los archivos municipales y de la documentación de los notarios públicos (cartórios públicos) descritos por Stein en el prefacio de la primera edición de su obra como "los más valiosos y quizás los repositorios más desatendidos por los historiadores, economistas, antropólogos y sociólogos (STEIN, 1985: IX).

4 "En Vassouras encontré, en el Archivo del Consejo Municipal, unas cuantas cajas con documentos bajo la escalera junto con pertenencias personales viejas y polvorientas. En la ciudad había tres notarios públicos, y tras haberlos visitado la decisión de estudiar esta comunidad fue prácticamente inmediata. Finalmente, Vassouras, una gran productora de café, tenía documentación abundante y hacía posible unas buenas condiciones para trabajar y para la vida familiar... Fue en este periodo cuando definí de forma más precisa mi intención de hacer un estudio comunitario de carácter socio-económico, prestando atención al devenir del café desde sus inicios hasta lo que consideré que sería el final de su presencia entre 1900 y $1910 \ldots$ Mi familia y yo nos mudamos rápidamente a Vassouras, donde, con solo unas breves interrupciones de viajes a Río de Janeiro para acabar la investigación, vivimos desde diciembre de 1948 a noviembre de 1949. Habiendo habitado ese largo periodo en Brasil puedo decir que me sentí bien adaptado e incluso parcialmente brasilianizado" (MEIHY, 1990: 85). 
La antropología también influyó en Stein de otra manera. Mientras planeaba el proyecto se interesó por la cuestión de "conseguir acceso a la 'voz' de los esclavos en las grandes plantaciones de café, recreando su mundo y particularmente encontrando formas de expresión de resistencia y adaptación" (STEIN, 2007: 39). Dos tipos de fuentes le sirvieron de inspiración para entrevistar a antiguos esclavos o descendientes de esclavos en Vassouras. Por un lado, los proyectos de entrevistas desarrollados en la década de 1930 en la Fisk University y por el Federal Writters' Project, una parte de los cuales habían sido publicados en el volumen Lay My Burden Down de Peter Botkin. Por otro, los estudios de Melville J. Herskovits y sus trabajos sobre la aculturación de los africanos en el 'nuevo mundo'. ${ }^{5}$ La importancia de la obra de Herskovits para el trabajo de Stein no sólo se limita a las perspectivas teóricas innovadoras que introdujo sobre las experiencias de los africanos en América, sino que también incluía nuevas técnicas de investigación.

Barbara Hadley Stein se encontró con Herskovits por vez primera en Bahía en 1941-1942 mientras realizaba su propia investigación. Antes de viajar a Vassouras, Stanley y Barbara Stein se reunieron con Melville Herskovits y su mujer Frances en la Northwestern University en Evanston, Illinois, para aprender algunas técnicas sobre las entrevistas orales. ${ }^{6}$ Durante esa visita Herskovits les mostró algunas grabaciones de música negra hechas en el curso de su trabajo de campo en el Caribe, en Brasil y en África. Esta experiencia hizo que Stanley Stein se diera cuenta de las posibilidades técnicas de recuperar voces y memorias (en este caso, una gran

5 "Un aprendiz de historiador social no podía dejar de estar estimulado por las nuevas perspectivas que planteaban los trabajos de Melville Herskovits sobre el proceso de aculturación y las experiencias de la población negra en general tanto en el Nuevo mundo como en África - The Myth of the Negro Past, Dahomey, and Trinidad Village (STEIN, 1990: xii).

6 "...cuando fuimos a Vassouras para recoger testimonios de la gente siempre recordaba lo que, en esencia, me dijeron. En primer lugar, que nunca tomara notas delante de las personas. Segundo, que siempre era yo quien debía de visitar a los informantes y hablar con ellos en su propio ambiente, no en el mío. Siguiendo estas sugerencias, después de haber hecho mis contactos, tuve el cuidado de ir a encontrarme con ellos en los campos y comencé a conversar. Preparé de antemano una larga serie de cuestiones $y$, con ellas en mente, fuimos a sus casas y hablábamos... Después de la entrevista me sentaba y transcribía mis notas. Luego las pasaba a máquina componiendo una especie de diario que después mi mujer organizaba por temas... Esto me proporcionó la base de información a partir de la cual reconstruir lo que había sido la vida de la población negra en tiempos de la esclavitud" (MEIHY, 1990: 87). 
y pesada grabadora prestada por la embajada de los Estados Unidos) que se plasmaron en sus grabaciones de jongos (LARA y PACHECO: 2007a). ${ }^{7}$ (En este contexto también debemos de llamar la atención sobre el uso que hizo Stein de la fotografía para documentar aspectos del trabajo y de la cultura material en el curso de su investigación en Vassouras). A partir de su contacto con Herskovits, Stein utiliza técnicas antropológicas para obtener documentación no escrita y ampliar así el abanico de fuentes puestas a disposición de los historiadores (STEIN, 2007: 39-40). La forma en que Stein concebía su proyecto combinaba el trabajo de campo antropológico con el trabajo en los archivos: "Establecí dos aproximaciones a la documentación: los datos registrados oficialmente y los informantes, detentadores de la memoria de los procesos. Creo que establecí una conexión entre los dos bloques de fuentes y así poder elaborar vínculos más pertinentes [entre ellos]" (MEIHY, 1990: 86).

\section{LOS COMMUNITY STUDIES Y LA SOCIEDAD DE PLANTACIÓN}

Los community studies se difundieron en la sociología norteamericana entre las décadas de 1920 y 1980, fundamentalmente por la importancia en el campo de las ciencias sociales de Robert Park y la Escuela de Chicago. Para el grupo que se formó alrededor de Park el concepto de "comunidad" era un instrumento para analizar el impacto de la modernización sobre las culturas rurales y urbanas. Era un concepto impreciso en tanto que la "comunidad" se refería en general a colectividades de pequeña escala -poblaciones rurales o enclaves urbanos, barrios o subculturas-. Frecuentemente se asociaba con aspectos positivos tales como solidaridad y lazos familiares y también como portadores de un propósito, interés e identidad comunes (RABINOWITZ, 2007: 2387).

En general hay dos aproximaciones teóricas a los community studies. La primera, quizás la más común actualmente, se basa sobre los sentimientos esenciales de identidad o de pertenencia, independientemente de la distribución geográfica de los miembros del grupo. El análisis de las "comunidades imaginadas" propuesto por Benedict Anderson (ANDERSON: 2008) ofrece un excelente ejemplo contemporáneo de este tipo de "sentimientos de comunidad". Este tipo de análisis es geográficamente difuso y ambiguo desde una perspectiva espacial. Incluye relaciones comunales extensas pero, en general, se limita a un conjunto de ideas singulares, valores y

7 Versos cantados espontáneamente en fiestas de esclavos (Caxambú). También algunas canciones de esclavos mientras trabajaban (STEIN, 1985: 297-298). 
creencias y no se plantea desde la complejidad. Es posible que comunidades sentimentales puedan existir en un espacio dado, pero normalmente se extiende más allá de lo local (p.e. nacionalismo, catolicismo, etc...). Desde este punto de vista las comunidades locales tienen poca importancia. Por otro lado, en un espacio concreto, aislado, único y homogéneo, las comunidades sentimentales no existen. Más bien coexisten múltiples comunidades, interactúan e incluso colisionan unas con otras (por no mencionar las condiciones de vida sociales y materiales que unifican la comunidad). Con este planteamiento los sentimientos colectivos se interpretan por medio de interacciones complejas que establecen contextos locales particulares y delimitados.

La segunda perspectiva de los community studies es más antigua pero también más habitual en el momento en que Stein escribió Vassouras. Con ella se enfatiza la base comunitaria y concibe a los grupos en un espacio definido. Esta óptica trata de entender la complejidad de las diversas relaciones y procesos que configuran una comunidad dada en el espacio como una totalidad (REDFIELD, 1989: esp. 1-16). Debemos mencionar que el concepto de "totalidad" no se refiere aquí a un conjunto empírico sino más bien a una construcción metodológica que puede ser conceptualizada desde diferentes perspectivas. Sin embargo, esta concepción llama la atención sobre la importancia de la formación de la comunidad ecológica y geográfica tanto como sobre las estructuras sociales, las relaciones y la organización que trasciende los sentimientos y los valores culturales. Todos estos elementos se conciben como parte de un todo y se analizan las relaciones entre ellos para reconstruir los contextos históricos relevantes. Además, esta perspectiva de los community studies proporciona un método para comprender las densas y específicas interconexiones de las relaciones que configuran la comunidad y no queda restringido al campo más amplio de los estudios regionales o nacionales.

Vassouras se elaboró de acuerdo con esta segunda concepción de comunidad. Sin embargo, el énfasis puesto en la unidad espacial, la solidaridad social y en la identidad de intereses y valores comunes-elementos inherentes en el propio concepto de comunidad-obligó a Stein a una reformulación para explicar el carácter histórico de su objeto de estudio. Para analizar el concejo de Vassouras como una comunidad Stein se enfrentó a tres problemas específicos. Primero tuvo que identificar los límites de una comunidad basada en una economía de monocultivo agroexportadora que dependía de la trata de esclavos africanos. En segundo lugar tuvo que dar cuenta de las desigualdades socioeconómicas y culturales, y de las divisiones y conflictos que caracterizaban una sociedad de plantación esclavista. Tercero, en el contexto de las divisiones existentes en la sociedad esclavista 
de Vassouras, tuvo que identificar los elementos y mecanismos que la integraban como comunidad.

Ante estos elementos heterogéneos, ambiguos y escasamente definidos de una sociedad de raíz esclavista basada en el monocultivo del café es importante resaltar aquí la influencia de Charles Wagley sobre el trabajo de Stein. ${ }^{8}$ En las propias palabras de Stein: "es importante recordar, sin embargo, que en 1947 yo estaba interesado en la economía y sociedad de plantación, no en la esclavitud en sí misma, y mucho menos en la historia comparada de la esclavitud o en la agricultura esclava en el Nuevo mundo. En otras palabras, mi atención se dirigía a lo que el antropólogo Charles Wagley denominó apropiadamente "plantation America" (STEIN, 2007: 36).

En el campo de los community studies Wagley fue uno de los responsables de la formulación y popularización del concepto "sociedad de plantación" como un sub-tipo de comunidad (WAGLEY: 1957; también MINTZ: 1953). Wagley concebía la plantación no como una unidad de producción sino como un tipo de sociedad. Identificando los aspectos característicos de la "sociedad de plantación" -monocultivo, una rígida división en clases, sociedades multirraciales, una débil estructura comunitaria, la presencia de pequeños campesinos propietarios y familias matrifocales- Wagley elaboró una tipología general para delimitar los elementos comunes de la "esfera cultural" de "Plantation America, que incluía el sur de los Estados Unidos, el Caribe y Brasil, extendiéndose desde Maryland hasta São Paulo. La propuesta de Wagley construyendo esta tipología era evitar investigaciones que se plantearan desde "términos excesivamente locales" y promover una perspectiva mucho más comparativa (WAGLEY, 1957: 11-12).9

\section{LA CONSTRUCCIÓN DE UN PROYECTO DE INVESTIGACIÓN}

Desde las concepciones antropológicas y sociológicas de los community studies, por un lado, y desde "plantation America", por el otro, Stein pudo ir más allá de un simple enfoque narrativo y construir un objeto de análisis mucho más complejo y multidimensional. Sin embargo, estos conceptos

8 Antropólogo y latinoamericanista especializada en Brasil, Charles Wagley fue profesor de antropología en Columbia University entre 1946 y 1971. Por casualidad Wagley y su esposa Cecilia estaban en el mismo barco en que Stanley y Barbara Stein viajaban a Brasil en 1948 para hacer su trabajo sobre Vassouras. Stein también conocía a la madre de Cecilia, Dona Belinha, e incluso se alojó en su casa en su primer viaje a Brasil en 1942 (MEIHY, 1990: 82 83). 
tipológicos son construcciones lógicas que se forman abstrayendo particularidades históricas para identificar características generales del modelo. Se conciben como si existieran fuera del tiempo y espacio histórico para crear las condiciones para la comparación (MINTZ, 1953: 136-143; REDFIELD: 1960). (El peligro de este tipo de planteamiento en que el carácter formalmente abstracto de las categorías rehúye de la vista y, a su vez, éstas son tomadas para representar distintas configuraciones históricas separadas unas de otras en el tiempo y en el espacio. La relación lógica entre modelos de comunidad se concibe, entonces, como si delimitara el actual curso del desarrollo histórico). Además, debido a las limitaciones que tales conceptos presentan para la investigación histórica, Stein debía de hacer frente al problema de reconstruir la "sociedad de plantación" de Vassouras como una comunidad histórica, configurada en unas relaciones y procesos específicos.

El interés de Stein por llevar adelante un proyecto de investigación sobre Vassouras estuvo motivado por su interés en la historia económica de Brasil y, por encima de todo, la historia del café. Como recientemente ha señalado: "Mi objetivo era analizar una cuestión que había estado presente en la historia de Brasil desde los comienzos del siglo XVI -los sucesivos ciclos de crecimiento orientados hacia la exportación-. Primero alrededor del azúcar, luego el tabaco, más tarde el oro y, desde el inicio del siglo XIX, el café (STEIN, 2007: 35). Este énfasis en la importancia tanto de los ciclos económicos como del café indica la influencia de los autores brasileños en el planteamiento de Stein. El cita la importancia de sus lecturas de Roberto Simonsen, Paulo Prado, Sergio Milliet, João Normano, Caio Prado Junior, los quince volúmenes de la historia del café de Affonso Tauney, así como los trabajos de Gilberto Freyre y las obras de los novelistas Monteiro Lobato y José Lins do Rego (STEIN, 1985 y 2007). No sería una exageración decir que la situación que atravesaba Brasil en la década de 1940 también era una influencia en el joven historiador: "Vassouras estuvo muy influenciada por la corriente nacionalista brasileña crítica con la continua agricultura de plantación agroexportadora con o sin trabajo esclavo, que había reducido

9 "Con el marco de la esfera cultural de Plantation-America existen innumerables 'variables' que hacen la comparación posible y alentadora ... Es precisamente desde esta proyección de la variación cultural -ya sea heredada desde Europa, derivada de las variaciones en el ambiente natural y sociocultural local o generada por distintas tendencias de desarrollo- frente al modelo común de la esfera cultural y buscando relaciones significantes en las que pudiéramos usar el método comparativo lo que nos ayudaría a construir una ciencia de la sociedad y la cultura" (WAGLEY, 1958: 12). 
a Brasil a la condición de país esencialmente agrícola y que obstaculizó la formación de una base industrial" (STEIN, 1985: XII-XIII). ${ }^{10}$

Con todas estas influencias Stein concibió su objeto de estudio del Valle de Paraíba haciendo uso del concepto de "ciclo del café". En sus propias palabras, "el completo desarrollo y declive de este ciclo, por los productores de café, podía ser percibido en un área geográfica, el valle medio y alto del Paraíba" (STEIN, 1985: xii). El ciclo del café -desde sus comienzos, pasando por su apogeo a mediados del Ochocientos, hasta su decadencia alrededor de 1900- establecía los límites temporales del periodo que analizó. A pesar de la importancia dada a este concepto del ciclo del café Stein rechazaba la posibilidad de escribir una historia general del café o de concebir la historia del Valle del Paraíba como una parte de la historia de Brasil o incluso como una simple historia "local" de una comunidad cafetalera. Por el contrario, optó por integrar la historia de una "sociedad de plantación" particular con la historia del ciclo del café en la segunda mitad del siglo XIX. "Barbara y yo", recordaba Stein recientemente, "discutimos sobre las diferentes opciones para desarrollar la investigación y decidimos analizar la sociedad cafetalera en un área específica de Brasil, analizándolo en la medida de lo posible con la esperanza de poder observarlo desde dentro y desde fuera" (STEIN, 2007: 36).

Sin embargo, el deseo de Stein de observar la comunidad "desde dentro y desde fuera" no era posible llevarlo a cabo sólo yuxtaponiendo "factores internos" y "factores externos". Más bien, inspirado por la escuela francesa de los Annales, el aspiraba a escribir una "historia total":

"Había una última influencia más a la hora de investigar y escribir Vassouras. Desde el principio tenía la intención, quizás demasiado ambigua, de elaborar un estudio holístico de una comunidad de plantación de café siguiendo las pautas de lo que entonces se denominaba "nueva historia": recrear un sentido de entorno tal como

10 "Llegué... a Brasil en 1949, en el punto álgido de la campaña "O Petróleo é Nosso". Era todo muy interesante, lleno de entusiasmo, dinamismo y contagioso!... Alimentaba mi deseo de saber más y entender mejor las cuestiones importantes del debate social, la vivacidad de aquella cultura... Lógicamente todos estos asuntos implicaban aspectos del debate sobre el papel de la agricultura, con lo que mi curiosidad iba en aumento. Fue un periodo en el que los efectos del pasado agrícola colonial podía ser medido y cualquiera podía reflexionar sobre la importancia y la relevancia de la agricultura en una fase que comenzaba con la industrialización moderna... Era una mosaico fresco y atractivo para alguien que venía de fuera con la intención de realizar estudios históricos que, de alguna forma, se cruzaban con todas estas cuestiones" (MEIHY, 1990: 85). 
lo haría un geógrafo humano integrando el suelo, la topografía, el clima y los hombres; detectar las redes de comunidad desde el prisma del antropólogo social; alcanzar una percepción de historiador de los factores y procesos a lo largo del tiempo" (STEIN, 1985: XII).

El proyecto de reconstruir la "historia total" de Vassouras en el ciclo del café del siglo XIX requería una investigación empírica, una reflexión metodológica y rigor teórico. Stein afirma: "esta definición temática, sin embargo, no era mecánica ni fácil. Por el contrario, siguió un devenir que combinaba mi desarrollo intelectual y mi preparación con las situaciones encontradas en el campo" (MEIHY, 1990: 84-85).

La ambición de Stein de escribir una "historia total", esto es, la visión simultánea de Vassouras "desde dentro y desde fuera", ponía de manifiesto el innovador carácter de su proyecto. Le obligaba a redefinir simultáneamente el concepto de ciclo económico y a historiar y especificar el concepto de "sociedad de plantación". Para lograrlo Stein rompió con la comprensión que sobre los ciclos dominaba en las ciencias sociales en Brasil. Los ciclos económicos son concebidos como entidades socio-económicas comprehensivas e integrales, no como la suma de sus partes. Se construyen a partir de la selección y elaboración de series de fenómenos, a menudo mediante operaciones estadísticas, como una función de su carácter repetitivo. El objetivo de esta operación es eliminar todas las variaciones accidentales y los factores alteradores para llegar a identificar relaciones espacio-temporales estables, homogéneas y "puras", y establecer relaciones causales entre ellas. Este procedimiento sólo reconoce lo que es homogéneo y comparable. Necesariamente ignora particularidades históricas y las concibe como una excepción particular. Tales excepciones deben tratarse como ejemplos de la relación general o como residuos accidentales y contingentes que no pueden tenerse en cuenta en el diseño del marco general (GINZBURG, 1993: 21; GRENIER y LAPETITE, 1989). En este mismo sentido, el concepto tipológico de "comunidad" se concibe como cerrado en sí mismo, una unidad integrada internamente que debe explicarse por el carácter y la configuración de sus propiedades internas. Considera los fenómenos externos a sus propios límites como entidades externas. Consecuentemente, para alcanzar la ambición de una historia "desde dentro y desde fuera" Stein tenía que ir más allá de lo que estas dos aproximaciones proponían sobre lo que era interno y lo que era externo, lo que era necesario y lo que era contingente para el análisis.

El carácter innovador de la contribución de Stein reside en su reconfiguración de los conceptos de "ciclo" y de "comunidad" mediante un análisis 
dialéctico de la relación entre los movimientos del mercado mundial y las fuerzas locales económicas, sociales y políticas que generaban la comunidad. Stein ve la "sociedad de plantación" de Vassouras como el resultado de procesos históricos específicos que operan a través de diferentes escalas espacio-temporales, no como algo dado de antemano. Por consiguiente la concibe como un complejo de relaciones mutuamente formativas más que como una configuración de factores separados e independientes. Desde este planteamiento Vassouras no se concibe como una comunidad encerrada en sí misma que es generada "desde fuera" por el ciclo del café y el mercado mundial, entendidos como "factores externos". Más bien, tomando la comunidad local como su punto de partida, Stein reconstituye los vínculos entre los procesos que operan en diferentes escalas espacio-temporales a través de las que la "sociedad de plantación" de Vassouras se forma.

"Para acercarse a la comunidad es suficiente con escoger un área representativa, analizarla desde una óptica interesante y que con una amplia perspectiva. Eso es lo que yo hice... El marco general vino de la inserción del análisis histórico, el pasado de la comunidad, en el contexto más amplio de la economía en una escala creciente, sugiriendo el pasaje de lo local a lo regional y de lo nacional a lo internacional... Era, por decirlo de alguna forma, lo contrario a lo que se estaba haciendo en Brasil donde las historias eran, casi siempre, historias generales" (MEIHY, 1990: 84-85).

Mediante este proceder metodológico el estudio especifica su objeto, la "sociedad de plantación de Vassouras" en el espacio y en el tiempo. Esto implica no sólo la reconstrucción de la comunidad sino también lo local como fenómeno histórico. Como se señala en el prefacio a la primera edición de Vassouras: "El objeto de este análisis de la economía y sociedad plantacionista de Vassouras, una comunidad en el Valle de Paraíba, en el centro-sur de Brasil, es examinar el efecto a nivel local de las transformaciones de la economía mundial sobre las instituciones brasileñas" (STEIN, 1985: VIII).

La relevancia y el valor analítico del proyecto de Stein arraigan en la reconstitución de las relaciones que integran la comunidad plantacionista de Vassouras con el ciclo del café y con los procesos históricos más amplios. En contraste con los posteriores estudios sobre la esclavitud, la plantación o la dependencia, que invariablemente privilegian una dimensión del complejo plantacionista -el mercado mundial, la producción esclavista, - la plantación- como su eje analítico principal y estrecha de este modo su enfoque, Stein nos presenta un concepto abierto de plantación que resulta útil para integrar la atención sobre diferentes relaciones -medioambientales, materiales, sociales, económicas, políticas y culturales-. De esta forma puede analizar los cambios en las interacciones históricas de estas 
relaciones a través del desarrollo histórico del complejo plantacionista de Vassouras, desde su fase embrionaria, pasando por su madurez, hasta su declive. Este método revela la compleja totalidad espacio-temporal de las relaciones y procesos históricos que constituyen la comunidad de Vassouras en el ciclo del café. Por un lado, Stein indica las particularidades de la comunidad de Vassouras en las más extensas redes de relaciones-locales, regionales, nacionales $y$, en el límite, globales-. Por otro, esta comunidad es un elemento específico en la formación de esas mismas redes, esclareciendo, desde este punto de vista, los procesos que configuran las escalas más amplias. Además Stein también establece no la tipicidad de Vassouras sino su especificidad en el ciclo del café. (Hemos de señalar que Vassouras es una comunidad cafetalera, pero no todas las comunidades cafetaleras son Vassouras).

\section{La arquitectura de Vassouras}

Tal como se ha planteado hay varias formas de pensar la comunidad como un todo. El enfoque de Stein sobre este aspecto es lo que determina la arquitectura de la obra, esto es, el modo de presentar el trabajo y su estructura analítica.

Stein comienza su análisis de Vassouras examinando la unidad ecológica de la comunidad. Establece esta unidad a partir de cómo se refleja el impacto del café en el medio ambiente y en la formación de la comunidad. Desde esta perspectiva enfatiza los aspectos físicos y materiales del cultivo del café en el contexto de la geografía particular del Valle y del enclave de Vassouras. Implícitamente distingue entre sistema ecológico y sistema productivo demostrando como la geografía específica y las condiciones medioambientales fueron transformadas con la introducción del café. Ya en la década de 1940 Stein mostraba su preocupación por la destrucción del bosque atlántico y por cómo la intensa explotación del medio ambiente derivaba en una migración de los sistemas agrícolas y en una mayor fragilidad de las bases de la prosperidad. Por otro lado, el análisis de la unidad ecológica-productiva de la comunidad cafetalera de Vassouras se complementaba con una reflexión sobre el mercado mundial. En este punto del análisis Stein destaca la creciente demanda europea y, sobre todo, estadounidense de café, la importación de mano de obra esclava y las fuentes y dinámicas del capital crediticio. De esta forma el análisis del desarrollo de la comunidad cafetalera de Vassouras queda enmarcado en condiciones y límites externos determinados por la naturaleza y por el mercado mundial.

Las condiciones materiales y los procesos de producción articulan el hilo 
organizativo del análisis de la unidad ecológico-productiva de Vassouras. Para establecer esta unidad y las características específicas de la comunidad Stein traza la expansión del cultivo del café en ese municipio y documenta el proceso de concentración de la tierra que conduce a la formación de las plantaciones cafetaleras que comenzaron en la década de 1820. Presta atención a las condiciones materiales, incluyendo la dimensión de las unidades de producción, los modelos de cultivo, los usos agrarios y las redes de transporte. De esta forma, además de realizar una cuidada descripción de la cultura material y de los aspectos arquitectónicos de las plantaciones nos muestra las rutinas y los ritmos de trabajo que estaban operativos en las plantaciones de café. Por último, interpreta los cambios en las condiciones de producción materiales y sociales como indicadores del crecimiento del cultivo cafetalero.

Desde la perspectiva de las condiciones materiales del cultivo de café Stein reconstruye la unidad social de la comunidad, su estructura específica formada por la diversificación y la reintegración de diferentes capas sociales que se relacionan las unas con las otras a través de la economía cafetalera y, al mismo tiempo, esas mismas capas son vistas como distintas e incluso en conflicto. La concentración de la tierra implica la marginación de aquellos que ocupan las tierras sin títulos, la dominación económica, social y política de los clanes de los grandes plantadores, y la aparición como un grupo social significativo de los poseedores que no debían de satisfacer renta alguna (agregados). En este contexto, Stein examina el tipo de trabajo demandado en las plantaciones de café y las diferentes categorías de trabajo existentes para llevarlo a cabo. A partir de aquí continúa analizando el impacto del comercio de esclavos tanto a nivel internacional como en el marco local con el objeto de valorar los cambios en la composición demográfica de la población esclava a lo largo del ciclo del café. Reconstruyendo la compleja y heterogénea estructura social de la comunidad Stein aborda la necesidad de establecer sus límites sociales. Con este propósito analiza la comercialización del producto, las redes de suministro local y las redes de transporte para identificar grupos como arrieros y agentes comisionistas que operaban en los márgenes de la comunidad pero que, a su vez, generaban un vínculo con el mundo exterior. De hecho, sus actividades serían lo que definiría la frontera social entre lo que estaba 'en' y lo que estaba 'fuera'. (Obviamente, la circulación de bienes, productos y crédito siguieron los circuitos nacionales e internacionales que se encontraban más allá de los límites de la comunidad). Tras distinguir los diferentes grupos por su rol económico Stein se acerca a la comunidad en términos de libres y esclavos, así como otras categorías sociales que de forma simultánea integraban y dividían la comunidad. 
De esta forma, sólo tras haber establecido la unidad del material diverso y los factores sociales Stein se encara a la relación entre amos y esclavos. Es importante considerar esta estrategia con cuidado porque, a pesar de la importancia de la relación amo-esclavo para determinar el carácter de la comunidad de Vassouras, ella por sí misma se configuraba en un complejo ya existente de relaciones y procesos históricos. Las acciones tanto de los amos como de los esclavos se formaron a través de unas condiciones materiales y unas relaciones sociales específicas. Fundando su análisis en condiciones históricas concretas Stein se orienta hacia la exploración de los temas clásicos de los estudios comunitarios desarrollados por los antropólogos. Así analiza las interrelaciones e interacciones entre amos y esclavos, reconstruye los modos de vida en las plantaciones además de las típicas biografías, valores y creencias. El camino que recorre revela la violencia de la dominación en la sociedad esclavista, las formas de resistencia esclava y las prácticas sociales y culturales que sostenían la integración de la comunidad a pesar de los antagonismos fundamentales existentes entre amos y esclavos y que, al límite, permitieron la formación de comunidades en el seno de la comunidad (REDFIELD, 1960: 113-131).

\section{Conclusión}

Basado en unos espacios y unas temporalidades específicas el Vassouras de Stanley Stein no es una historia general (MINTZ, 1959: 557); y al mismo tiempo es "algo más que una historia local" (WAGLEY, 1958: 420). La obra nos ofrece un amplio abanico de datos y perspectivas $y$, por esta misma razón, queda abierta a varias lecturas posibles. Como reflejo de los cambios de interés y perspectiva a lo largo de los últimos cincuenta años, el libro ha sido leído como una crítica de la interpretación de Gilberto Freyre sobre el pasado esclavista brasileño, como una contribución al análisis de la dependencia económica brasileña, como una fuente para una historia comparada entre el Valle de Paraíba y la región occidental de São Paulo, como un modelo metodológico para una historia local agraria, y como una interpretación, modelo de investigación y fuente de información para una cultura y una memoria de la población esclavizada (LARA, 2007b: 53-67).

Cada una de estas lecturas encuentra una base legítima en el libro de Stein. Sin embargo, todas ellas resultan parciales. Quisiera plantear que el gran valor de su obra radica en su "aprendizaje de una historia total", esto es, en su reconstrucción de los procesos y relaciones materiales, sociales y culturales que configuraron una sociedad de plantación esclavista en el ciclo del café a lo largo del siglo XIX. La riqueza de su análisis desde esta totalidad centrada en la comunidad de Vassouras permite toda esa serie 
de lecturas parciales. Sin embargo, lo que resulta más importante es lo que nos revela en lo que se refiere a la interrelación entre los fenómenos históricos que no son concebidos de forma aislada.

Desde la perspectiva que propone Stein cada proceso histórico tiene lugar en un amplio complejo de relaciones que es lo que configura las comunidades e interactúa con otros procesos. La densidad de todo ese abanico de interdependencias e interacciones es lo que estructura el contexto específico de los procesos históricos y haciendo así más profunda su comprensión. Recordando las palabras del historiador británico E.P. Thompson "la historia es tanto una disciplina de contextos como de procesos: cada significado es un significado en su propio contexto, y las estructuras cambian mientras las viejas formas pueden expresar nuevas funciones $y$ las viejas funciones pueden encontrar su expresión bajo nuevas formas" (THOMPSON, 1977: 256). El enfoque metodológico de Stein ofrece de forma clara un recorrido interpretativo en un conjunto histórico dinámico.

Sin embargo, me gustaría mencionar para concluir otros dos aspectos que se encuentran implícitos en la obra de Stein que quizás resultan relevantes para los estudios históricos contemporáneos -los que tienen que ver con la representación y con la escala-. Para Stein, como para los antropólogos, el valor de los estudios de las comunidades locales es lo que representan como un 'microcosmos' en el que se observan procesos más generales (STEIN, 2007: 37-38; REDFIELD, 1960: 103, 105). A modo de reflejo de mundos más extensos el enfoque de Stein sobre la comunidad posibilita la realización de análisis más concretos que son más cercanos a la experiencia vivida y a la acción humana de lo que generalmente podemos realizar desde los estudios macrohistóricos. De todas formas resulta necesario prestar atención a la contribución de la microhistoria italiana. Como ha subrayado Giovanni Levi, no existen mecanismos automáticos a partir de los cuales los actores sociales se alinean con cambios y transformaciones estructurales. Estos procesos presentan problemas para los análisis históricos. Siguiendo a Levi, la reducción de la escala representa un método analítico y experimental capaz de revelarnos factores que previamente habían pasado desapercibidos. Mediante este proceso la microhistoria busca descubrir anticipadamente sucesos anómalos o inadvertidos que ponen de relieve las incoherencias en un orden aparentemente unificado (LEVI, 1991: 107). ${ }^{11}$ De esta forma quienes se dedican a la microhistoria abren

11 Sin embargo, incluso la extrema reducción en la escala llevada a cabo por los microhistoriadores -ya sea un pueblo, un grupo de familias o un individuo, acontecimiento u objeto- de 
un espacio a la reflexión sobre las comunidades en contextos mucho más amplios.

Por otro lado, podemos interpretar Vassouras desde una perspectiva opuesta. El enfoque de Stein es la comunidad que quiere analizarla "desde dentro y desde fuera". A pesar de su reconfiguración del concepto de 'ciclo', su noción de fuera permanece como una concepción abstracta y general del mercado mundial. Cambiando la unidad de análisis a la economía mundo llega a ser viable considerar la comunidad plantacionista como el objeto de análisis en el marco de procesos más amplios. Así, resulta posible situar la comunidad local plantacionista de Vassouras en los procesos de expansión de la economía mundo y de la reconfiguración de la geografía mundial y la división económica del trabajo.

Con esta perspectiva, resultan evidentes las similitudes entre la región cafetalera del Valle de Paraíba, el territorio algodonera del sur de los Estados Unidos y la zona azucarera en Cuba. Desde este punto de vista, el análisis de Vassouras nos revela no la repetición lineal de un sistema esclavista arcaico y un régimen plantacionista amenazado por la modernidad económica, social y política sino, más bien, la formación de un nuevo espacio productivo en el que la reconstitución del sistema esclavista y de la economía de plantación tienen lugar en el marco de un mercado mundial integrado y en una evidente división del trabajo. Todo ello caracterizado por la expansión de la producción industrial, por la independencia política de las antiguas colonias europeas en el Nuevo Mundo y por la ascensión de las ideologías liberales -en las que se incluyen tanto el abolicionismo nacional como internacional-. En otras palabras: la creación de una nueva frontera de la esclavitud. Con este planteamiento, el Vassouras de Stein tiene todavía mucho que enseñarnos.

Traducción: Vicent Sanz Rozalén

forma inevitable deja un residuo de lo que Ginzburg describe como un "margen especulativo irrefrenable" (GINZBURG, 1992: 96-125, esp. 105-107). Aunque los microhistoriadores han ampliado el abanico de posibilidades explicativas e interpretativas, lo microhistórico no es más "real" que otros niveles de análisis espacio-temporales (TOMICH, 2012: 28-29). 


\section{Bibliografía}

ANDERSON, B. (1983): Imagined Communities: Reflections on the Origins and Spread of Nationalism. Verso, Londres [trad. cast. (1993): Comunidades imaginadas. Reflexiones sobre el origen y la difusión del nacionalismo. FCE, Madrid].

BOTKIN, B.A. (ed.) (1945): Lay My Burden Down: A Folk History of Slavery. University of Chicago Press, Chicago.

GINZBURG, C. (1993): "Microhistory: Two or Three Things That I Know About It". Critical Inquiry, n' 20/1, pp. $10-35$ [trad. cast. (1994): "Microhistoria. Dos o tres cosas que sé de ella". Manuscrits, $n^{\circ} 12$, pp. 13-42]. GRENIER, J.-Y. y LEPETIT, B. (1989): "L'expérience historique: Sur C.-E. Labrousse". Annales E.S.C., n44/6, pp. 1337-1360.

LARA, S.H. y PACHECO, G. (orgs.) (2007a): Memória do Jongo. As gravações de Stanley J. Stein. Vassouras, 1949. Folha Seca/ CECULT, Río de Janeiro/São Paulo.

LARA, S.H. (2007b): "Vassouras e os sons do cativeiro no Brasil", en LARA, S.H. y PACHECO, G. (orgs.). Memória do Jongo, pp. 45-67.

LE RIVEREND, J. (1959): Reseña de Vassouras. A Brazilian Coffee County, 18501900 [Stanley J. Stein]. Revista de Historia de América, n47, pp. 293-294.

LEVI, G. (1991): "On Microhistory", en BURKE, P. (ed.), New Perspectives on Historical Writing. Polity Press, Cambridge, pp. 93-1 13 [trad. cast. (1999): "Sobre microhistoria", en BURKE, P: Formas de hacer historia. Alianza, Madrid].

MEIHY, J.C.S.B. (1990): "Como um todo a comunidade brasilienista não se mostrou passiva... Entrevista com Stanley J. Stein", A colônia brasilianista: história oral de vida acadêmica. Nova Stella, São Paulo, pp. 79-101.

MINTZ, S.W. (1959): Reseña de Vassouras. A Brazilian Coffee County, 1850-1900 [Stanley J. Stein]. The Economic History Review, New Series, vol. $11 / 3$, p. 557.

MINTZ, S.W. (1953): "The Folk-Urban Continuum and the Rural Proletarian Community". American Journal of Sociology, n59/2, pp. 136-143.

RABINOWITZ, D. (2001): "Community Studies: Anthropological", en International Encyclopedia of the Social \& Behavioral Sciences. Elsevier, Amsterdam, vol. 4, pp. 2387-2389.

REDFIELD, R. (1960): Little Community and Peasant Society and Culture. University of Chicago Press, Chicago (reed. 1989, Midway).

STEIN, S.J. (1957): Vassouras: A Brazilian Coffee County, 1850-1900. Harvard UP, Cambridge. 
STEIN, S.J. (1961): Grandeza e Decadência do Café. Brasiliense, São Paulo. STEIN, S.J. (1970): Vassouras: A Brazilian Coffee County, 1850-1900. Atheneum, Nueva York.

STEIN, S.J. (1985): Vassouras: A Brazilian Coffee County, 1850-1900. The Roles of Planter and Slave in a Plantation Society. Princeton UP, Princeton. STEIN, S.J. (1990): Vassouras: Um município brasileiro do café, 18501900. Editora Nova Fronteira, Río de Janeiro.

STEIN, S.J. (2007): "Uma viagem maravilhosa", en LARA, S.H. y PACHECO, G. (orgs.), Memória do Jongo, pp. 35-42.

THOMPSON, E.P. (1977): "Folklore, Anthropology, and Social History". The Indian Historical Review, $\mathrm{n}^{\circ} 3 / 2$, pp. 247-266 [trad. cast. (1989): "Folklore, antropología e historia social". Historia Social, $n^{\circ} 3$, pp. 81 102].

TOMICH, D.W. (2012): "The Order of Historical Time: The Longue Durée and Micro-History", en LEE, R.E. (ed.): The Longue Durée and World-Systems Analysis. SUNY Press, Albany.

TOMICH, D. (ed.) (2016): New Frontiers of Slavery. SUNY Press, Albany. WAGLEY, Ch. (1957): "Plantation America: A Cultural Sphere", en RUBIN, V. (ed.), Caribbean Studies: A Symposium. University of Washington Press, Seattle, pp. 3-13.

WAGLEY, Ch. (1958): Reseña de Vassouras. A Brazilian Coffee County, 1850-1900 [Stanley J. Stein]. The Hispanic American Historical Review, vol.38/3, pp. 420-422. 